\section{Diverse clinical phenotypes associated with a nonsense mutation in FAM161A}

\begin{abstract}
Purpose: Mutations in the FAM161A gene have been reported in association with autosomal recessive retinitis pigmentosa (arRP) in several ethnic populations. This study aimed to assess the prevalence of FAM161A-related retinopathy in a British cohort and to characterise the phenotype associated with mutations in this gene. Methods: The FAM161A coding region and intron-exon boundaries were screened by Sanger sequencing in $\mathbf{1 2 0}$ retinitis pigmentosa (RP) patients (with likely autosomal recessive inheritance) in whom mutations in other known major RP genes have been ruled out by commercially available testing. Homozygosity mapping was performed in one consanguineous family, and high-throughput sequencing of candidate genes was performed to identify disease-associated changes. Clinical assessment of affected individuals included perimetry testing, fundus autofluorescence imaging, and optical coherence tomography.
\end{abstract}

Results: Two patients of British origin with a homozygous mutation in FAM161A (c.1309A > T, p.Arg437*) were identified by Sanger sequencing. Homozygosity mapping and subsequent high-throughput sequencing analysis identified a further family of Pakistani origin with the same genotype. Clinical examination of affected members of these families revealed that this mutation was associated with a diverse clinical phenotype, ranging from mild disease with preservation of central acuity to severe visual impairment. Conclusions: Homozygosity for the c.1309A > T, p.Arg437* variant in FAM161A is a relatively common cause of arRP. The mutation occurs in diverse ethnic populations, associated with typical retinitis pigmentosa with disease onset usually in the second or third decade of life.

Eye (2015) 29, 1226-1232; doi:10.1038/eye.2015.93; published online 26 June 2015
AM Rose1, P Sergouniotis ${ }^{1,2}$, G Alfano', N Muspratt-Tucker ${ }^{1}$, S Barton ${ }^{2}$, AT Moore ${ }^{1,3}$, G Black², SS Bhattacharya' and AR Webster ${ }^{1,3}$

Introduction

Biallelic mutations in the FAM161A gene have been reported as a rare cause of autosomal recessive retinitis pigmentosa $(\mathrm{RP})$ in several ethnic populations including Indian, Middle Eastern, North African, and German. ${ }^{1-3}$ The gene is expressed in several tissues including the heart, brain, liver, lung, kidney, and muscle, but expression is particularly elevated in the retina. In the murine retina, FAM161A localises to photoreceptor cells during development, and to the inner segment of photoreceptor cells and outer plexiform layer in the adult retina. FAM161A is a ciliary protein, and thus FAM161A-associated disease can be considered a retinal ciliopathy. ${ }^{4}$

FAM161A spans $29 \mathrm{~kb}$ of genomic DNA at chromosome 2p15 and comprises seven coding exons. The gene encodes two major isoforms, formed by alternative splicing of exon 4 (exon numbering as per NCBI RefSeq ID NM_001201543.1). The major isoform of the protein is formed of 660 amino acids (NCBI RefSeq ID NM_032180.2), whereas the less common isoform (formed by retention of exon 4; NCBI RefSeq ID NM_001201543.1) comprises 716 amino acids. ${ }^{2}$ Both isoforms contain a 277-amino-acid domain (UPF0564) that mediates microtubule association ${ }^{5}$ and has been previously identified in at least 15 other human proteins. The UPF0564 domain contains three conserved coiled-coil motifs that, in other proteins, mediate oligomerisation and proteinprotein interactions. The FAM161A protein has been shown to interact with several other proteins (POC1B, SDCCAG8, lebercilin, OFD1, CEP290); mutations within these genes lead to retinal degenerations or multi-system disease. Mutations in POC1B have been associated with cone-rod retinal dystrophy, ${ }^{5}$ and mutations in LCA5 - which encodes lebercilin-have been associated with Leber's congenital amaurosis. ${ }^{4}$ A minority of Bardet-Biedl syndrome cases are caused by mutations in SDCCAG8; this 
syndrome presents with retinal dystrophy, learning difficulties, renal malformation, obesity, and polydactyly. ${ }^{4}$ Joubert syndrome is a multisystem disorder that is characterised by cerebellar malformation and RP; FAM161A was shown to interact with two Joubert syndrome-associated proteins, OFD1 and CEP290, the latter also being associated with Leber's congenital amaurosis. ${ }^{4}$

Although it has been shown that FAM161A is required for routing of outer segment proteins, such as opsin and rds/peripherin 2, its precise function remains unclear. 6,7

We aimed to gain insights into the frequency of FAM161A mutations in patients with RP seen at a single UK centre and to characterise the retinal phenotype in individuals with FAM161A-related disease.

\section{Materials and methods}

A total of 120 unrelated patients with a clinical diagnosis of RP and a family history suggestive of autosomal recessive inheritance were recruited. The inclusion criteria were a confirmed diagnosis of RP with neither parent affected by disease; with or without consanguinity and/or other affected siblings. These inclusion criteria will unavoidably include some patients with X-linked or de novo autosomal dominant disease. All study subjects presented to the inherited eye disease clinics of a tertiary referral centre (Moorfields Eye Hospital, London, UK) and had no known molecular diagnosis. All patients had undergone commercial genetic testing of major retinal degeneration loci, including dominant, recessive, and syndromic forms of RP (Manchester Regional Genetic Laboratory Services). At the time of testing, the commercially available panel did not include RP28/FAM161A.

Genomic DNA was isolated from peripheral blood leukocytes under standard conditions. PCR primers (Sigma-Aldrich, Haverhill, UK) were designed to amplify each exon and exon-intron junctions of the major transcript NM032180.2 (sequences available upon request). PCR was performed using GoTaq Colourless Master Mix 2X (6.4 $\mu \mathrm{l}$, Promega), distilled water $(6.4 \mu \mathrm{l})$, forward/reverse primer (60 $\mathrm{nM}$ each), and $10 \mathrm{ng}$ of DNA. The reaction was denatured at $95^{\circ} \mathrm{C}(10 \mathrm{~min})$, and 35 cycles of amplification (30 s at $95^{\circ} \mathrm{C}, 30 \mathrm{~s}$ at $55^{\circ} \mathrm{C}, 45 \mathrm{~s}$ at $72^{\circ} \mathrm{C}$ ) were performed, before a final extension step $\left(72^{\circ} \mathrm{C}, 10 \mathrm{~min}\right)$. The PCR products were purified using ExoSAP-IT (USB), before sequencing with BigDye v3.1 (Applied Biosystems, Life Technologies Ltd, Paisley, UK) under standard conditions on an ABI3730 DNA sequencer.

In addition to these 120 subjects, a sibling pair of Pakistani origin (family GC3796) was recruited. The two affected brothers were born to consanguineous parents and have been previously tested using single-nucleotide polymorphism (SNP) chip arrays (GeneChip Human Mapping $50 \mathrm{~K}$ Xba Array, Affymetrix) according to the manufacturer's recommendations. The pedigree was consistent with the propagation of a single mutant allele from a recent ancestor such that affected individuals were autozygous for this allele and homozygosity mapping was performed using a custom-made python script interacting with a MySQL database. ${ }^{8}$ High-throughput next-generation sequence analysis was subsequently performed to identify the exact disease-causing variant using an Agilent SureSelect capture (Custom Design, enriching for 105 known retinal dystrophy genes) $)^{9}$ and an ABI SOLiD 5500XL sequencer. Sequence data were mapped with SOLiD Lifescope software (Life Technologies Ltd) with the hg19 human genome as a reference. Variants were called using a combination of the Lifescope software suite and Samtools (http://samtools. sourceforge.net/), and filtered for those SNPs with $\geq 5 \times$ coverage. Using Ensembl V.68, variants were annotated to genes, and functional consequences were determined. Likely disease-associated variants that were identified by this method were confirmed by Sanger sequencing.

\section{Results}

Sequencing of the FAM161A gene in 120 subjects with RP revealed individuals of British origin (GC18398, GC19423) harbouring a homozygous variant in the gene (c.1309A > T, p.Arg437*); they were not knowingly related. Homozygosity mapping of an additional family with an affected sibling pair (family GC3796; Pakistani origin) revealed four large regions of homozygosity, including a 29-Mb region on chromosome 2 that encompassed the FAM161A gene (Table 1). Subsequent high-throughput sequencing of retinal disease-associated genes revealed the same FAM161A mutation (c.1309A > T, p.Arg437*), again in the homozygous state.

Haplotype analysis was performed across the FAM161A coding region for SNPs with minor allele

Table 1 Regions of extended homozygosity identified in a consanguineous Pakistani family (GC3796), including the FAM161A locus

\begin{tabular}{|c|c|c|c|c|c|}
\hline Chromosome & Contiguous SNPs & Start & Finish & Size $(b p)$ & Genomic region and genes of interest \\
\hline 2 & 723 & 45834000 & 75622000 & 29788000 & 2p21-13.1 FAM161A, fibulin3, ALMS1 \\
\hline 8 & 119 & 126018000 & 130437000 & 4419000 & $8 \mathrm{q} 24$ No candidates \\
\hline 10 & 298 & 69403000 & 86443000 & 17040000 & 10q21.3-23.1 CDH23, RGR, PCDH21 \\
\hline 22 & 189 & 15268000 & 26688000 & 11420000 & 22q11-12 TIMP3 \\
\hline
\end{tabular}


frequency $>0.01$, in order to ascertain whether c. $1309 \mathrm{~A}>\mathrm{T}$ is a founder mutation in the Eurasian population (Table 2). This demonstrated that the two individuals of British origin (GC18398 and GC19423) had the same FAM161A haplotype. Given the SNP allele frequencies, it was estimated that the probability of the mutation arising de novo on the same haplotype was $2.1 \%$, and it was thus concluded that this change represents a founder mutation in the European population. The haplotype of the Pakistani sibling pair (GC3796) was, however, different from that observed in the British individuals.

\section{Clinical history of GC18398}

Subject GC18398, a 52-year-old white British woman, was diagnosed with RP in her 20s, after reporting problems with her peripheral vision. Since the diagnosis there has been progressive visual fields loss and the development of nyctalopia. Past ophthalmic history included bilateral cataract extraction at the age of 40 years. At the last examination, at the age of 49 years, central visual acuity was relatively well preserved (6/12 each eye), but there was significant limitation of the visual fields (constricted to $10^{\circ}$ eccentricity in each eye). Fundoscopy revealed a small amount of retinal pigment migration in the right and left eyes (Figure 1a and b). Fundus autofluorescence (FAF) imaging revealed an abnormal peripheral retina and a hyperautofluorescent ring around the fovea (Figure 1c and d). The patient has no siblings and neither parent was affected by disease; there was no history of consanguinity (Figure 1e).

\section{Clinical history of GC19423}

Subject GC17745, a 41-year-old white British woman, was diagnosed with RP at the age of 15 years. The patient has one affected sister, and the parents are first cousins (Figure 2a). Since adolescence, there has been a progressive loss in peripheral visual field and problems with vision in dim illumination. At the last examination, at the age of 40 years, corrected visual was 6/9 in each eye. There was severe bilateral visual field loss $\left(<5^{\circ}\right.$ in each eye). Slit lamp examination showed mild posterior subcapsular lens opactities. Fundal examination revealed extensive retinal pigment migration with more nummular pigment deposits in the peripheral retina (Figure $2 b$ ). FAF imaging showed a perifoveal ring of high density and reduced autofluorescencent signal in the periphery in each eye (Figure $2 \mathrm{c}$ and $\mathrm{d}$ ). Optical coherence tomography (OCT) showed moderate thinning of the central retina in each eye (Figure 2e and f).

\section{Clinical history of two individuals from family GC3796}

Family GC3796 is a consanguineous Pakistani family, with three affected family members; 2 affected brothers were examined: subject IV.1 and subject IV.2 (Figure 3a).

Subject IV.1 is a 48-year-old man who was diagnosed with RP at the age of 31 years, but despite this late presentation clinical progression of disease has been relatively fast. He had undergone right cataract surgery at the age of 42 years. At the age of 45 years, visual acuity was $6 / 36$ in both eyes, and at his most recent examination, at the age of 48 years, vision was hand movements bilaterally; there was severe visual field loss $\left(<5^{\circ}\right.$ in each eye). Fundoscopy revealed extensive retinal pigment migration and attenuation of the retinal vasculature with optic disc pallor (Figure 3b). FAF imaging showed loss of peripheral autofluorescence in each eye, with normal fluorescence pattern at the central macula (Figure 3c). OCT revealed preservation of retinal layers at the fovea, although cystic changes were noted in each eye in the perifoveal region (Figure $3 \mathrm{~d}$ and e).

Subject IV.2 is a 46-year-old man who was diagnosed with RP at the age of 26 years and, as with his sibling, clinical progression of disease has been relatively fast. He had undergone bilateral cataract surgery at the age of 45 years. At the age of 41 years, visual acuity was 6/60 in both eyes, and at the most recent examination, at the age of 45 years, vision was reduced to perception of light in both eyes. Fundus examination showed signs of advanced RP (Figure 3f). FAF imaging and OCT were not performed in this individual.

Table 2 Haplotype analysis of coding region SNPs in FAM161A

\begin{tabular}{llccccc}
\hline & Alleles & MAF & Exon & GC18398 & GC19423 & GC3796 \\
\hline rs4270331 & C/A & 0.02 & 1 & C,C & C,C & C,C \\
rs11125895 & T/C & 0.15 & 2 & C,C & C,C & C,C \\
rs144455077 & G/A & 0.01 & Splice site & G,G & G,G & G,G \\
rs17513722 & T/C & 0.15 & 3 & C,C & C,C & T,T \\
rs149314387 & C/T & 0.01 & 3 & C,C & C,C & C,C \\
rs4672457 & G/A & 0.02 & 3 & G,G & G,G & G,G \\
rs77562614 & C/T & 0.01 & 6 & C,C & C,C & C,C \\
\hline
\end{tabular}

The grey shading indicates where individuals GC18398 and GC19423 carried the minor allele in homozygous state. 

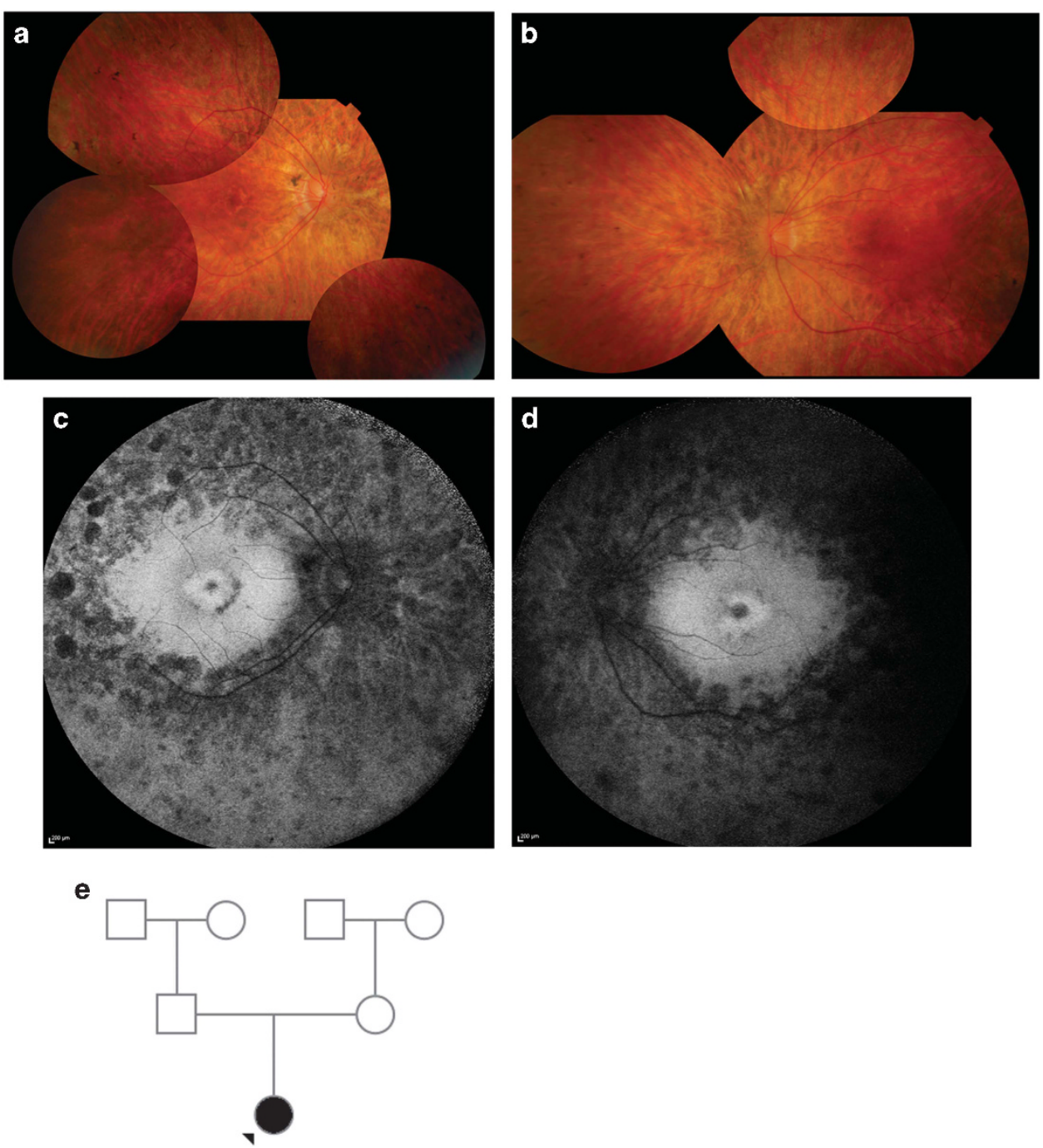

Figure 1 Clinical findings in individual GC18398. Fundoscopy revealed peripheral changes in the right and left eye, with minimal bone spicule pigmentation in the inferonasal and superotemporal aspects of the retina $(a, b)$. FAF imaging demonstrated extensive loss of autofluorescence in the peripheral retina and a parafoveal ring of high density in both eyes (c, d). Family pedigree of the patients (e).

\section{Discussion}

This study aimed to assess whether mutations in FAM161A caused recessive RP in a cohort of patients from a single UK referral centre, and to characterise the phenotype associated with mutations in this gene. We screened a panel of 120 probands with recessive RP, and two were found to harbour biallelic FAM161A variants. This panel was enriched for rare causes of RP, as it comprised patients in whom previous molecular genetic testing had been negative. Our results are not dissimilar to findings from previous studies in North American and German populations in which FAM161A mutations accounted for $\sim 1 \%$ of recessive RP cases. ${ }^{4,10}$ Notably, a higher disease frequency has been described in the Israeli and Palestinian populations. ${ }^{3}$
In our study, three unrelated families harbouring the same FAM161A nonsense mutation (c.1309A > T, p. $\operatorname{Arg} 437^{*}$ ) in the homozygous state were identified. Two of the families carrying the mutation were of British origin, with the remaining family originating from Pakistan. The same mutation (c.1309A > T, p.Arg437*) has been previously reported in a homozygous state in three apparently unrelated German individuals with FAM161A-related disease. ${ }^{4}$

Affected members of the two British families (GC18398 and GC19423) had an identical haplotype across the entire coding region of FAM161A, and it is likely that the mutation is a founder mutation in the British population. The haplotype of the British patients was, however, different from that of three seemingly unrelated German patients who carried the same 
mutation (but who shared the same intragenic haplotype to each other). ${ }^{4}$ It seems, then, that the mutation has arisen de novo in two distinct European populations. Furthermore, the same mutation was observed in family GC3796, who are of Pakistani origin and carried a different haplotype. It seems likely, therefore, that the c.1309A > T change occurs at a mutation hotspot, therefore arising de novo in distinct, unrelated populations.

We have not excluded the possibility that any of these individuals carry this mutation in the hemizygous state-with a complete deletion of one copy of FAM161A on the second chromosome. The region surrounding
FAM161A on chromosome 2 is rich in short interspersed elements, such as Alu repeats, which can cause a deletion of large genomic regions through the process of nonallelic homologous recombination.

All four FAM161A-positive individuals had identical mutations, but there were some differences in the clinical findings. Subjects GC19423 and GC18398 had typical teenage-onset RP with minimal retinal pigmentation and a hyperautofluorescent ring on FAF imaging. The two individuals from family GC3796 had adult-onset disease but with surprisingly rapid deterioration. There was no hyperautofluorescent ring on FAF imaging in one of the two siblings who was available for testing.
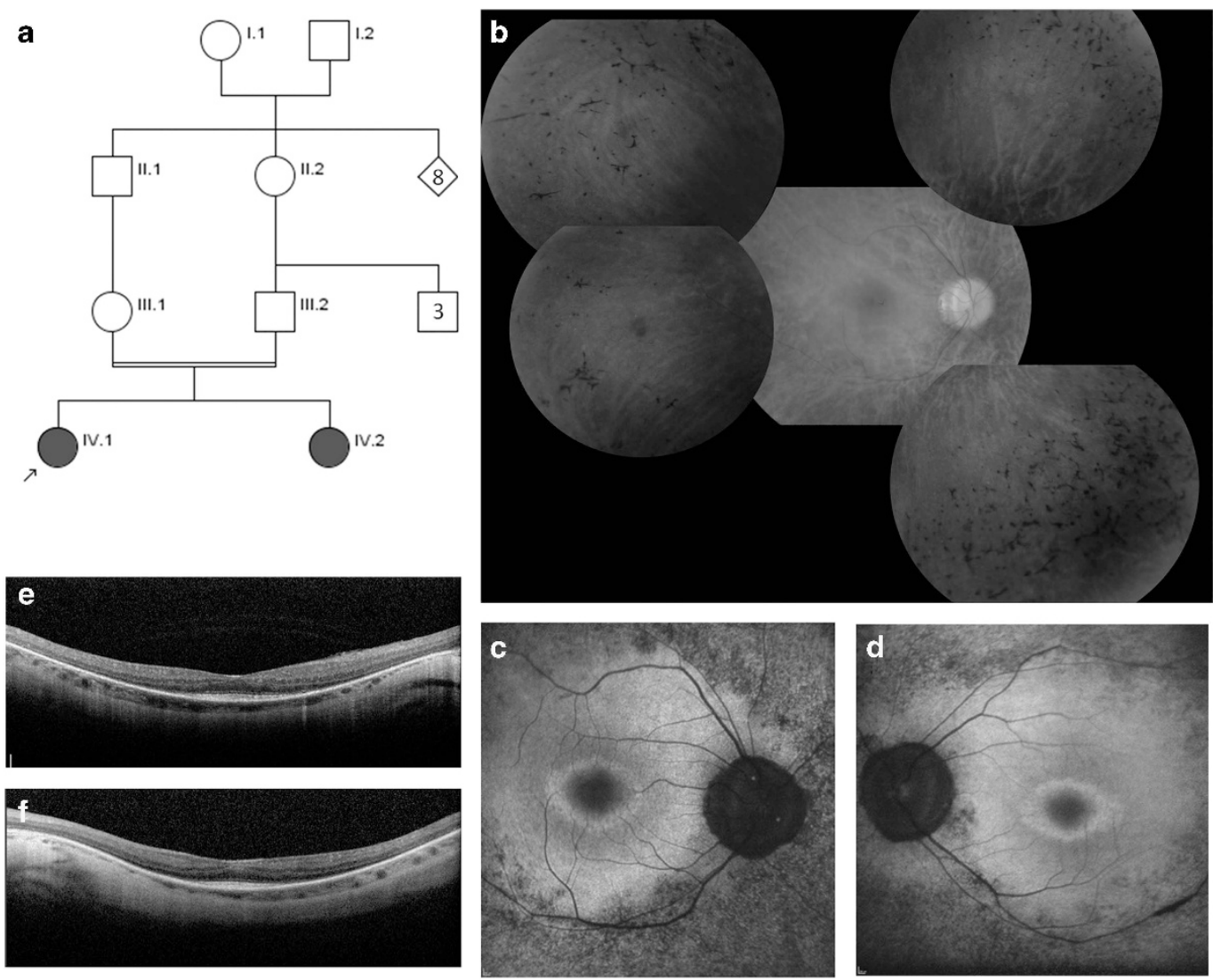

Figure 2 Clinical findings in individual GC19423. Family pedigree showing consanguinity: the patient is indicated (a). Fundoscopy of the right eye revealed slight pigmentation in the peripheral retina that was most prominent in the superonasal aspect (b); similar changes were observed in the left eye (not shown). FAF imaging demonstrated loss of autofluorescence in the peripheral retina and a parafoveal ring of high density in right eye (c) and left eye (d). OCT demonstrated preserved retinal layers in the foveola only (e, f).

Figure 3 Clinical findings in family GC3796. Family pedigree showing consanguinity: the two patients are indicated (a). In individual IV.1, fundoscopy of the right eye revealed pigmentation in the peripheral retina, including nummular deposits-most prominent in the temporal and inferior aspects (b); similar changes were observed in the left eye. FAF imaging in individual IV.1 demonstrated a loss of autofluorescence in the peripheral retina in the right eye (c) and left eye (not shown). OCT demonstrated some preservation of foveal architecure in the right eye (d) and left eye (not shown); a few areas consistent with macular oedema can be observed in the right eye (e) and also in the left eye (not shown). Fundoscopic appearances of individual IV.2 were similar to that of IV.1, with extensive peripheral pigment deposition, pallor of the optic disc, and attenuation of the retinal vessels (f); symmetrical changes were observed in the left eye (not shown). 
a
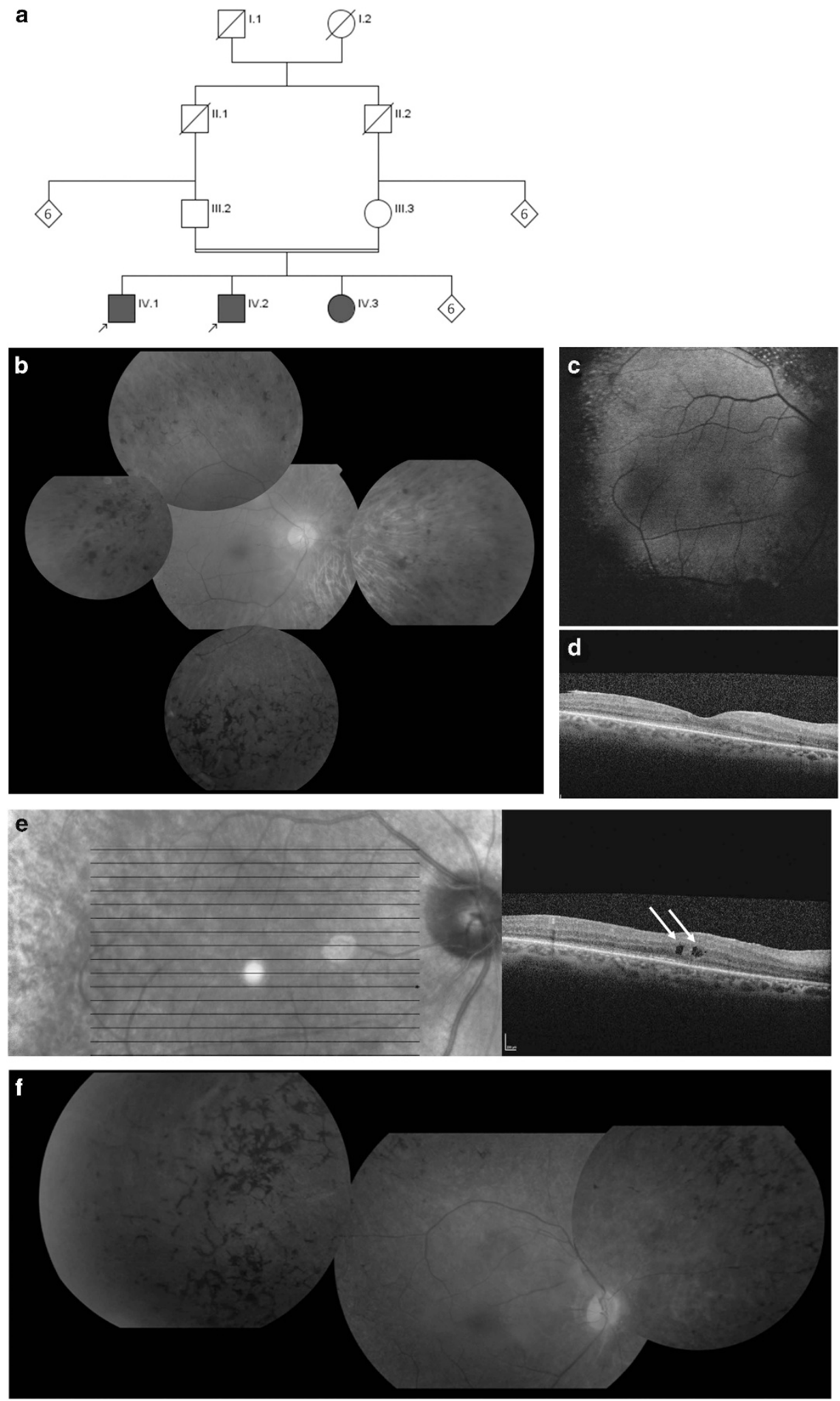
These results are consistent with previous studies describing variability in age of onset and rate of disease progression. ${ }^{3,4,11,12}$ The limited degree of intraretinal pigment migration and a ring of increased signal on FAF imaging are frequent features of FAM161A retinopathy. $3,4,11,12$

In summary, mutations in FAM161A are a relatively common cause of autosomal recessive retinitis pigmentosain patients with RP seen at a UK referral centre; all patients with identified mutations had the same c.1309A > T, p.Arg437* mutation in FAM161A, and the presence of this mutation in different ethnic groups suggests that it may occur at a mutational hotspot in the gene.

\section{Summary}

\section{What was known before}

- Mutations in FAM161A are associated with autosomal recessive retinitis pigmentosa.

- The c.1309A > T, p.Arg437* mutation in FAM161A has been observed in German patients with autosomal recessive retinitis pigmentosa.

What this study adds

- The p.Arg437* mutation was observed in British autosomal recessive retinitis pigmentosa subjects. Although seemingly unrelated, the mutation was on a common haplotype, suggesting that the mutation is a founder in the British population.

- The p.Arg437* mutation was observed in a Pakistani family on a different haplotype, suggesting that this change occurs at a mutation hotspot.

- The four individuals harbouring p.Arg437* mutation had strikingly different clinical phenotypes, suggesting the existence of genetic modifiers of disease.

\section{Conflict of interest}

The authors declare no conflict of interest.

\section{Acknowledgements}

The authors gratefully acknowledge funding from The Rosetrees Foundation, RP Fighting Blindness, Moorfields Special Trustees, NIHR Biomedical Research Centre at Moorfields Eye Hospital, and Foundation Fighting Blindness, USA. The authors also gratefully acknowledge bioinformatic analyses of the high-throughput sequencing data by Simon Williams and experimental assistance from Dr Amna Shah and Dr Wendy Mustill.

\section{References}

1 Gu S, Kumaramanickavel G, Srikumari CR, Denton MJ, Gal A. Autosomal recessive retinitis pigmentosa locus maps between D2S1337 and D2S286 on chromosome 2p11-p16 in an Indian family. J Med Genet 1999; 36: 705-707.

2 Bandah-Rozenfeld D, Mizrahi-Meissonnier L, Farhy C, Obolensky A, Chowers I, Pe'er J et al. Homozygosity mapping reveals null mutations in FAM161A as a cause of autosomal-recessive retinitis pigmentosa. Am J Hum Genet 2010; 87: 382-391.

3 Langmann T, Di Gioia SA, Rau I, Stöhr H, Maksimovic NS, Corbo JC et al. Nonsense mutations in FAM161A cause RP28associated recessive retinitis pigmentosa. Am J Hum Genet 2010; 87: 376-381.

4 Di Gioia SA, Letteboer SJ, Kostic C, Bandah-Rozenfeld D, Hetterschijt L, Sharon D et al. FAM161A, associated with retinitis pigmentosa, is a component of the cilia-basal body complex and interacts with proteins involved in ciliopathies. Hum Mol Genet 2012; 21(23): 5174-5184.

5 Roosing S, Lamers IJ, de Vrieze E, van den Born LI, Lambertus S, Arts HH et al. Disruption of the basal body protein POC1B results in autosomal-recessive cone-rod dystrophy. Am J Hum Genet 2014; 95(2): 131-142.

6 Zach F, Grassmann F, Langmann T, Sorusch N, Wolfrum U, Stöhr H. The retinitis pigmentosa 28 protein FAM161A is a novel ciliary protein involved in intermolecular protein interaction and microtubule association. Hum Mol Genet 2012; 21(21): 4573-4586.

7 Karlstetter M, Sorusch N, Caramoy A, Dannhausen K, Aslanidis A, Fauser S et al. Disruption of the retinitis pigmentosa 28 gene Fam161a in mice affects photoreceptor ciliary structure and leads to progressive retinal degeneration. Hum Mol Genet 2014; 23(19): 5197-5210.

8 Sergouniotis PI, Davidson AE, Mackay DS, Li Z, Yang X, Plagnol V et al. Recessive mutations in KCNJ13, encoding an inwardly rectifying potassium channel subunit, cause leber congenital amaurosis. Am J Hum Genet 2011; 89(1): 183-190.

9 O'Sullivan J, Mullaney BG, Bhaskar SS, Dickerson JE, Hall G, O'Grady A et al. A paradigm shift in the delivery of services for diagnosis of inherited retinal disease. J Med Genet 2012; 49 (5): 322-326.

10 Duncan JL, Biswas P, Kozak I, Navani M, Syed R, Soudry S et al. Ocular Phenotype of a Family with FAM161Aassociated Retinal Degeneration. Ophthalmic Genet 2014; 9: 1-9 (e-pub ahead of print).

11 Zobor D, Balousha G, Baumann B, Wissinger B. Homozygosity mapping reveals new nonsense mutation in the FAM161A gene causing autosomal recessive retinitis pigmentosa in a Palestinian family. Mol Vis 2014; 20: 178-182.

12 Venturini G, Di Gioia SA, Harper S, Weigel-DiFranco C, Rivolta C, Berson EL. Molecular genetics of FAM161A in North American patients with early-onset retinitis pigmentosa. PLoS One 2014; 9(3): e92479. 